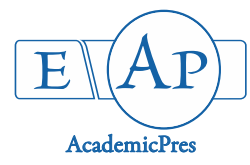

\title{
Carbapenem-Resistant Enterobacteriaceae among In-Patients of Tertiary Hospitals in Southwest, Nigeria
}

\author{
Ibikunle I. ANIBIJUWON, Ifeoluwa D. GBALA*, Olusoji O. ADEBISI \\ University of Ilorin, Faculty of Life Sciences, Department of Microbiology, Ilorin, \\ Nigeria; ifeoluwagbala@yahoo.com (*orrespondingauthor)
}

\begin{abstract}
The present study assessed the incidence and risk factor associated with the occurrence of carbapenem-resistant Enterobacteriaceae (CRE) among hospitalized patients at three tertiary hospitals in Southwest, Nigeria. A cross-sectional study was performed over a six-month surveillance period in the locations with a total of 300 blood and urine samples. A structured close-ended questionnaire was also administered to all subjects for review of demographics and potential risk factors. Fifty-nine isolates belonging to genera Escherichia (52.5\%), Enterobacter (23.8\%), Klebsiella (10.2\%) and Proteus (13.6\%) were isolated. All the isolates were multi-drug resistant with a notable resistance (100\%) to cephalosporins and significant sensitivity to nitrofurantoin. A total of 23 isolates including Escherichia coli $(\mathrm{n}=8)$, Enterobacter aerogenes $(\mathrm{n}=9)$, Klebsiella pneumoniae $(\mathrm{n}=1)$ and Proteus mirabilis $(\mathrm{n}=5)$ exhibited resistance to one or both of imipenem and meropenem. The overall incidence of CRE in the three locations at the time of study was $7.7 \%$. Age $(p=0.01)$ and exposure to invasive devices were significant risk factors for CRE colonization. Although at low incidence, the occurrence of CRE among this group calls for active monitoring because of its implication fatality of infections as well as the propensity to spread.
\end{abstract}

Keywords: carbapenem; carbapenem-resistant; enterobacteriaceae; in-patients; multi-drug resistance

\section{Introduction}

The increase in the rate of antimicrobial resistance exhibited by bacteria, especially the Enterobacteriaceae family, is a threat to public health (Albiger et al., 2015). This threat decreases the ability to successfully treat numerous infectious diseases, while simultaneously increasing health risks for vulnerable patients. Carbapenemresistant Enterobacteriaceae (CRE) are able to inactivate carbapenems which are revered to possess higher antibacterial spectrum against Gram negative bacteria than penicillin, cephalosporins and other $\beta$-lactams (ECDC, 2013; Jeong et al., 2015).

CRE produce seriously difficult to treat infections (urinary tract infections, septicemia, pneumonia, or intraabdominal infections) in debilitated and immunecompromised patients, in association with prolonged hospitalization and increased fatality, with mortality rates up to $50 \%$ (Guh et al., 2015). Few studies have been carried out on CPE strains in Nigeria, with reports of incidence ranging between 10-33.5\% (Yusuf et al., 2012a, 2012b; Ejikeugwu et al., 2013; Motayo et al., 2013; Mohammed et al., 2015; Oduyebo et al., 2015). Although carbapenems are unusually prescribed in Nigerian hospitals, there still remains a necessity for a focused approach on the dissemination of CRE. Also, CRE has the disposition to spread quite promptly, especially in an hospital environment where the risk of transmission is much higher; therefore a low incidence is not to be ignored, but rather a charge to curb further spread.

The objective of the present study was to determine the occurrence, frequency of distribution and risk factors associated with the colonization of CRE in three tertiary hospitals situated in Southwest Nigeria.

\section{Materials and Methods}

\section{Description of study area}

The study was conducted in three states in the Southwestern part of Nigeria. The region's population is predominantly heterogenous with people of varied religious and ethnic beliefs. The study was carried out in three tertiary hospitals from Ekiti, Osun and Oyo states (Fig. 1).

\section{Study population and description}

The study was carried out among all consenting age groups (children, adults and aged) who were hospitalized and also met the inclusion criteria in the selected hospitals that were surveyed. The survey/ sample collection was carried out in all three locations from October 2016 to March 2017. 


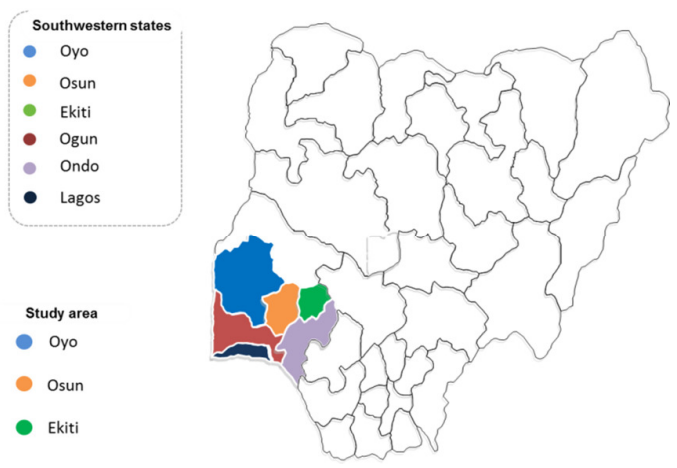

Fig. 1. Map showing study areas

Inclusion criteria for CRE screening

- Patients who had been hospitalized within 3 weeks and above

- Patients who undergone surgery

- Patients with urinary tract infections

- Patients with invasive devices such as catheter

- All age groups who met the aforementioned criteria

\section{Ethical consideration}

Ethical approval for the research was obtained foremost from the Ethics Review Board of the Faculty of Life Sciences, University of Ilorin. Then, approval was obtained from the Ethics Review Committee of the selected hospitals after which informed consent was obtained from the subjects and/or their caretakers.

\section{Data collection}

Questionnaires were administered to all subjects who participated in the study. The response to the questionnaires was used to collate information about the socio-demographic characteristics such as age, gender and socioeconomic status (level of education and occupation) of the participants. The questionnaire also provided information on the patients' medical history, use of antibiotics and patients' exposures to the risk factors associated with the colonization of CRE.

\section{Sample collection}

Blood or urine samples were obtained from subjects who met the inclusion criteria, placed in an ice chest and transported to the laboratory for analysis.

\section{Procedure for isolation of Enterobacteriaceae}

Blood sample: Following the collection of the blood samples directly into brain heart infusion broth (Rapid Labs, UK), the samples were incubated for 5 days at $37^{\circ} \mathrm{C}$. After 5 days, the blood culture was subcultured by streaking a loopful of the culture suspension on solidified MacConkey and Blood agars (Oxoid, UK) and incubated at $37^{\circ} \mathrm{C}$ for $24-48$ hours.

Urine sample: the samples obtained were analyzed within 6 hours of collection. A loopful of urine was aseptically picked from the urine sample and streaked on a solidified CLED (Lab M, UK) agar; then incubated at 37 ${ }^{\circ} \mathrm{C}$ for 24 hours.
Identification of isolates

The isolates were identified by using the $\mathrm{Hi} 25^{\mathrm{TM}}$ Enterobacteriaceae identification kit (HiMedia Laboratories Ltd., India) and PCR.

\section{Antibiotic susceptibility test}

Assay for the antibiogram pattern of the isolates was carried out using the disc diffusion method as described by Bauer et al. (1985). The antibiotics used (Rapid labs, UK and Oxoid, UK) and their corresponding concentrations are as follows: Ceftazidime $(30 \mu \mathrm{g})$, Cefuroxime $(30 \mu \mathrm{g})$, Gentamicin $(10 \mu \mathrm{g})$, Ciprofloxacin $(5 \mu \mathrm{g})$, Ampicillin (10 $\mu \mathrm{g})$, Ofloxacin $(5 \mu \mathrm{g})$, Augmentin $(30 \mu \mathrm{g})$, Nitrofurantoin $(300 \mu \mathrm{g})$, Imipenem $(10 \mu \mathrm{g})$ and Meropenem $(10 \mu \mathrm{g})$.

\section{Determination of susceptibility to antibiotics}

Solidified Mueller Hinton agar (Rapid Lab, UK) plates were seeded with $100 \mu$ l of the standardized organisms and were spread evenly over the total surface area of the agar using a glass spreader. Multiple antibiotics discs containing eight of the aforementioned antibiotics were carefully and firmly placed on the surface of the agar using sterile forceps. For imipenem and meropenem, single discs of each of the antibiotics were placed. Afterwards, the plates were incubated at $37^{\circ} \mathrm{C}$ for $18-20$ hours. After incubation, the zones of inhibition generated by the antibiotics were measured. The values for each organism against the antibiotics were interpreted using the breakpoints interpretative criteria of Clinical and Laboratory Standard Institutes (CLSI, 2016).

\section{Statistical analysis}

The descriptive statistics of the data obtained in the study was analyzed and/ or plotted using SPSS 22.0, SigmaPlot. The odd ratio was calculated using MEDCALC and Vassarstats online statistical software to measure the association of variables as risk factor in CRE colonization. The $p$-value for significance was at $\mathrm{p} \leq 0.05$.

\section{Results}

Demographic characteristics and risk factor assessment

All age groups specified for the study participated in all three hospitals except at Oyo state where none of the subjects was older than 60 years. Equal ratio of the genders was used in all the three locations (Table 1). Over $80 \%$ of the subjects have been on hospitalization within 3 weeks in the three hospitals, while 14-18\% were hospitalized for over 3 weeks. Also, more of the participants in the three hospitals (66\%, 88\% and $89 \%$ respectively) had no recent surgical procedures. On the evaluation of antibiotics use, it was gathered that most of the participants in the three hospitals were on antibiotics within $1-3$ weeks (64\%, $78 \%$ and $75 \%$ ). A similar trend of $21 \%$ or $22 \%$ was recorded in all locations for use of antibiotics from 3 weeks to 3 months. Exposure to catheter or other invasive devices during hospitalization in the three hospitals ranged between $17-42 \%$. Subjects with previous diagnosis of UTI ranged from $28-34 \%$ in the hospitals, while records of previous hospitalization were < $40 \%$ (Table 2). 
312

\section{Isolation of Enterobacteriaceae}

In summary, a total of $34(57.6 \%)$ of all fifty-nine (59) isolates obtained in the present study were recovered from urine samples, while 25 (42.4\%) were from blood samples. Thirty-one (52.5\%) of the isolates were Escherichia coli, 14 (23.8\%) were Enterobacter aerogenes, 6 (10.2\%) were Klebsiella sp. and 8 (13.6\%) belonged to Proteus sp. (Table 3).

\section{Antibiotic susceptibility test}

Isolates that showed multiple resistances to one to five antibiotics were categorized as multi-drug resistant (MDR); resistance to six to nine antibiotics was categorized as extensive drug resistance (XDR), while complete resistance to all antibiotics was categorized as pan-drug resistance (PDR). In TE, out of the 21 isolates tested, $28.6 \%$ exhibited MDR, while $71.4 \%$ were XDR. All the isolates showed complete resistance to augmentin, ampicillin, ceftazidime and cefuroxime. Resistance to other antibiotics was as follow: ciprofloxacin (61.9\%), gentamicin (57.1\%), ofloxacin (57.1\%), nitrofurantoin (19\%), imipenem (19\%) and meropenem (19\%) (Fig. 2a). Notably, there was a fairly low level of resistance against the carbapenems (imipenem and meropenem), with four of the twenty-one isolates being resistant (Fig. 3).

In TOY, all the isolates were completely resistant to augmentin, ceftazidime and cefuroxime. The resistant pattern to other antibiotics include- ampicillin (95\%), ciprofloxacin (50\%), gentamicin (40\%), ofloxacin $(40 \%)$, nitrofurantoin (30\%), meropenem (30\%) and imipenem $(20 \%)$ (Fig . 2b). Four (4) and six (6) isolates out of the total 20, showed resistance to imipenem and meropenem, respectively (Fig. 3). In TOS, 15 isolates (83.3\%) exhibited $\mathrm{XDR}$ with resistance to at least six antibiotics while $11.1 \%$ showed MDR (Fig. 2c). Also, PDR was observed in one of the Enterobacter aerogenes isolates. All the isolates were completely resistant to ampicillin, ceftazidime and cefuroxime.

Resistance to other antibiotics include- augmentin (94.4\%), gentamicin (83.3\%), ciprofloxacin (83.3\%), ofloxacin (72.2\%), meropenem (50\%), imipenem (33.3\%) and nitrofurantoin (16.7\%). Six (6) and nine (9) out of 18 isolates were resistant to imipenem and meropenem, respectively (Fig. 3). A total of 23 isolates exhibited resistance to one or both of imipenem and meropenem in all the three locations.

Table 1. Demographic characteristics of the subjects used in the study

\begin{tabular}{|c|c|c|c|}
\hline \multirow{2}{*}{ Characteristics } & \multicolumn{3}{|c|}{ Frequency and percentage of respondents ${ }^{*}$} \\
\hline & $\mathrm{TE}$ & TOY & TOS \\
\hline \multicolumn{4}{|c|}{ Age } \\
\hline $6-10$ & 5 & 8 & 7 \\
\hline $11-20$ & 13 & 13 & 7 \\
\hline $21-30$ & 14 & 35 & 27 \\
\hline $31-40$ & 32 & 22 & 17 \\
\hline $41-50$ & 12 & 16 & 18 \\
\hline $51-60$ & 11 & 6 & 12 \\
\hline $61-70$ & 10 & 0 & 1 \\
\hline $70+$ & 3 & 0 & 11 \\
\hline \multicolumn{4}{|c|}{ Gender } \\
\hline Female & 50 & 50 & 50 \\
\hline Male & 50 & 50 & 50 \\
\hline \multicolumn{4}{|c|}{ Type of education } \\
\hline Formal & 54 & 70 & 64 \\
\hline Informal & 46 & 30 & 36 \\
\hline \multicolumn{4}{|c|}{ Marital status } \\
\hline Single & 29 & 36 & 27 \\
\hline Married & 66 & 62 & 70 \\
\hline Widowed & 3 & 1 & 2 \\
\hline Divorced & 2 & 1 & 1 \\
\hline \multicolumn{4}{|c|}{ Occupation } \\
\hline Top civil servant/ executive & 18 & 24 & 23 \\
\hline Middle class/ small scale business & 10 & 9 & 13 \\
\hline Junior staff/ petty trader & 13 & 28 & 31 \\
\hline Artisan & 42 & 13 & 11 \\
\hline Others & 17 & 26 & 22 \\
\hline
\end{tabular}


Table 2. Risk factors evaluated among subjects

\begin{tabular}{|c|c|c|c|}
\hline \multirow{2}{*}{ Characteristics } & \multicolumn{3}{|c|}{ Percentage of respondents ${ }^{*}$} \\
\hline & TE & TOY & TOS \\
\hline \multicolumn{4}{|c|}{ Duration of hospitalization } \\
\hline Within 3 weeks & 82 & 86 & 83 \\
\hline More than 3 weeks & 18 & 14 & 17 \\
\hline \multicolumn{4}{|c|}{ Recent surgical procedure } \\
\hline Yes & 34 & 12 & 11 \\
\hline No & 66 & 88 & 89 \\
\hline \multicolumn{4}{|c|}{ Duration of antibiotics use } \\
\hline 1 - 3 weeks & 64 & 78 & 75 \\
\hline 3 weeks -3 months & 22 & 21 & 22 \\
\hline 3 months + & 14 & 1 & 3 \\
\hline \multicolumn{4}{|c|}{ Exposure to invasive devices } \\
\hline Yes & 42 & 17 & 30 \\
\hline No & 58 & 83 & 70 \\
\hline \multicolumn{4}{|c|}{ Quality of hospital hygiene } \\
\hline Poor & 4 & 1 & 1 \\
\hline Fair & 21 & 14 & 9 \\
\hline Good & 45 & 41 & 41 \\
\hline Very good & 20 & 24 & 26 \\
\hline Excellent & 10 & 20 & 23 \\
\hline \multicolumn{4}{|c|}{ Response to treatment } \\
\hline Poor & 2 & 0 & 0 \\
\hline Fair & 13 & 3 & 5 \\
\hline Good & 54 & 61 & 57 \\
\hline Excellent & 31 & 36 & 38 \\
\hline \multicolumn{4}{|c|}{ Previous diagnosis of UTI } \\
\hline Yes & 28 & 34 & 31 \\
\hline No & 72 & 66 & 69 \\
\hline \multicolumn{4}{|c|}{ Previous hospitalization } \\
\hline Yes & 39 & 31 & 36 \\
\hline No & 61 & 69 & 64 \\
\hline
\end{tabular}

Table 3. Summary of isolates obtained from the three study sites

\begin{tabular}{cccccc}
\hline \multirow{2}{*}{ Sample } & \multirow{2}{*}{ No. of isolates/ Proportion (\%) } & \multicolumn{4}{c}{ Isolate distribution } \\
\cline { 3 - 6 } & & Escherichia coli & Enterobacter aerogenes & Klebsiella sp. & Proteus sp. \\
\hline Urine & $34(57.6)$ & $21(35.6)$ & $7(11.9)$ & 0 & $6(10.2)$ \\
Blood & $25(42.4)$ & $10(16.9)$ & $7(11.9)$ & $6(10.2)$ & $2(3.4)$ \\
Total & $59(100)$ & $31(52.5)$ & $14(23.8)$ & $6(10.2)$ & $8(13.6)$
\end{tabular}

Table 4. Univariate analysis of the risk factors in association with CRE colonization

\begin{tabular}{|c|c|c|c|c|c|c|c|}
\hline \multirow{2}{*}{ S/N } & \multirow{2}{*}{ Variable } & & \multicolumn{2}{|c|}{ CRE } & \multirow{2}{*}{ p-value } & \multirow{2}{*}{ Odd ratio } & \multirow{2}{*}{$95 \% \mathrm{CI}$} \\
\hline & & & Positive & Negative & & & \\
\hline \multirow[t]{2}{*}{1.} & Age & $21-50$ & 13 & 180 & 0.01 & 0.32 & $0.13-0.77$ \\
\hline & & $51-70$ & 10 & 44 & & & \\
\hline \multirow[t]{2}{*}{2.} & Gender & Male & 14 & 136 & 0.28 & 1.61 & $0.68-3.85$ \\
\hline & & Female & 9 & 141 & & & \\
\hline \multirow[t]{2}{*}{3.} & Duration of hospitalization & $\leq 3$ weeks & 16 & 235 & 0.06 & 0.41 & $0.16-1.05$ \\
\hline & & $>3$ weeks & 7 & 42 & & & \\
\hline \multirow[t]{2}{*}{4.} & Nature of illness & Infectious & 10 & 148 & 0.36 & 0.67 & $0.28-1.58$ \\
\hline & & Non-infectious & 13 & 129 & & & \\
\hline \multirow[t]{2}{*}{5.} & Recent surgical procedure & Yes & 3 & 54 & 0.45 & 0.62 & $0.18-2.16$ \\
\hline & & No & 20 & 223 & & & \\
\hline \multirow[t]{2}{*}{6.} & Duration of antibiotic use & $\leq 3$ weeks & 13 & 204 & 0.08 & 0.47 & $0.20-1.11$ \\
\hline & & $>3$ weeks & 10 & 73 & & & \\
\hline \multirow[t]{2}{*}{7.} & Exposure to invasive devices & Yes & 11 & 78 & 0.05 & 2.34 & $0.99-5.52$ \\
\hline & & No & 12 & 199 & & & \\
\hline \multirow[t]{2}{*}{8.} & Previous hospitalization & Yes & 12 & 94 & 0.08 & 2.12 & $0.90-4.99$ \\
\hline & & No & 11 & 183 & & & \\
\hline
\end{tabular}

CI: Confidence Interval Significance $(\mathrm{p} \leq 0.05)$ 

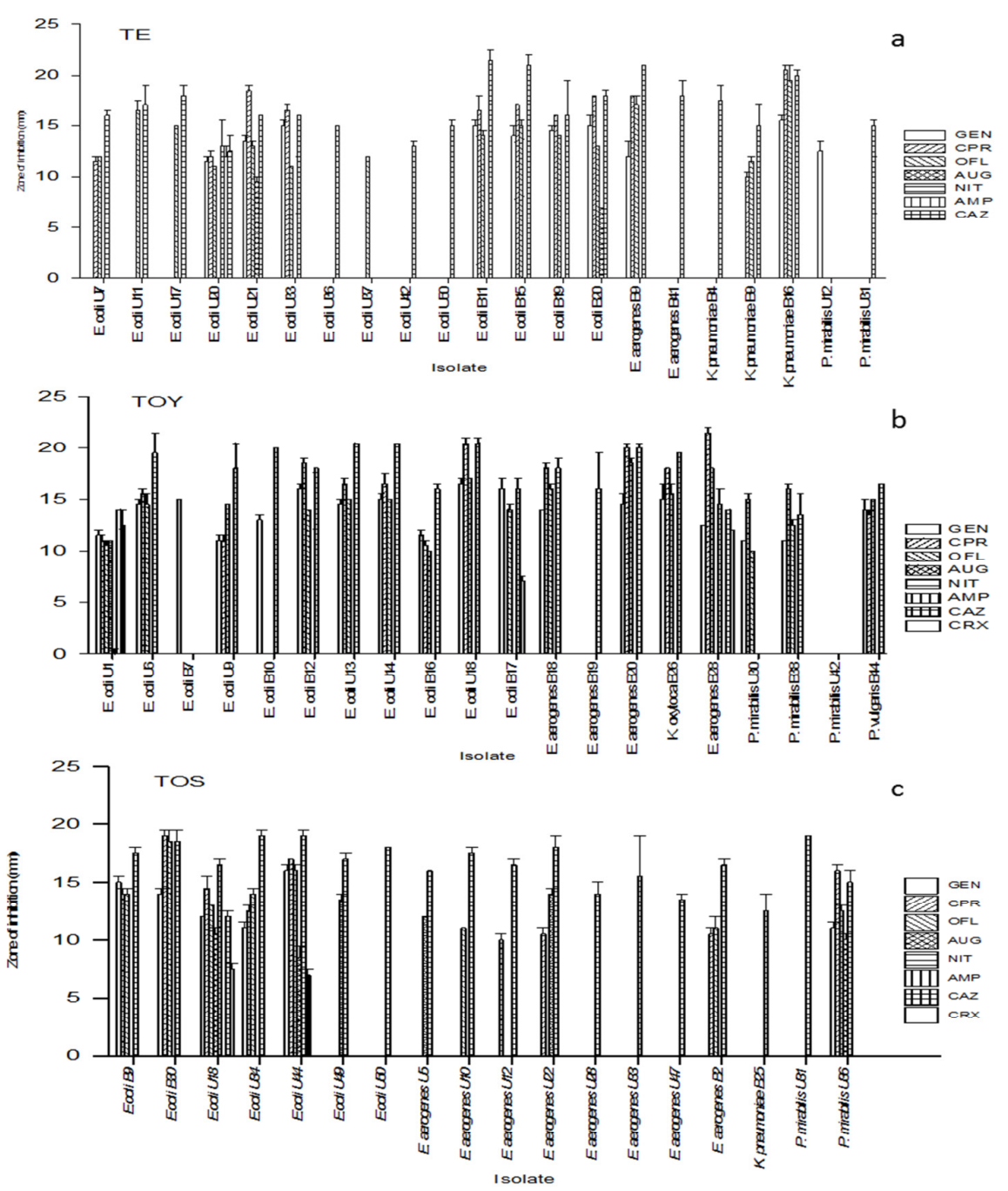

Fig. 2. Antibiotic susceptibility pattern of the isolates from the three locations

\section{Incidence proportion and risk factor association}

The incidence proportion of CRE in TE was 6\%, while the incidence rate was 3 cases per 100 people; in TOY, 7\% with an incident rate of 3.5 cases per 100 people; in TOS, $10 \%$, while incident rate was 5 cases per 100 people. Age ( $\mathrm{p}$ $=0.01)$ and exposure to invasive devices $(\mathrm{p}=0.05)$ were significantly associated with CRE colonization. The duration of hospitalization $(\mathrm{p}=0.06)$, prolonged use of antibiotics $(\mathrm{p}=0.08)$ and previous hospitalization $(\mathrm{p}=$ 0.08 ) were also associated with CRE colonization.

The odds of CRE colonization was 68\% higher in patients of ages 51 years and above. Patients who had been on hospitalization for over 3 weeks had $59 \%$ higher odds of CRE colonization compared to patients who were on hospitalization within 3 weeks (Table 4). Gender was not a confounding factor in CRE colonization $(\mathrm{p}=0.28)$ although odds of CRE colonization was $16.1 \%$ lower in males. Presence of underlying medical condition $(p=36)$ and recent surgical procedures $(\mathrm{p}=45)$ were also not associated with CRE colonization (Table 4).

\section{Discussion}

\section{Demographic characteristics of the subjects}

Over $50 \%$ of the participants in each location belonged to the age group $21-50$ years old; less than $10 \%$ were aged 610 years old and $\leq 11 \%$ were 70 years and above. For the present study, a higher number of the participants were adults (21-50 years) and thus may be considered to be a mixed population of individuals at the peak of their immunity, as well as those with a probable decline in immune system efficacy. The gender of the respondents was evenly distributed in all the locations. This was done in order to critically assess the possible influence of gender on colonization or infection by CRE.

\section{Isolation of Enterobacteriaceae}

Enterobacteriaceae are known to be the most representative bacteria family in the cause of nosocomial infections and outbreaks (Cai et al., 2017). 

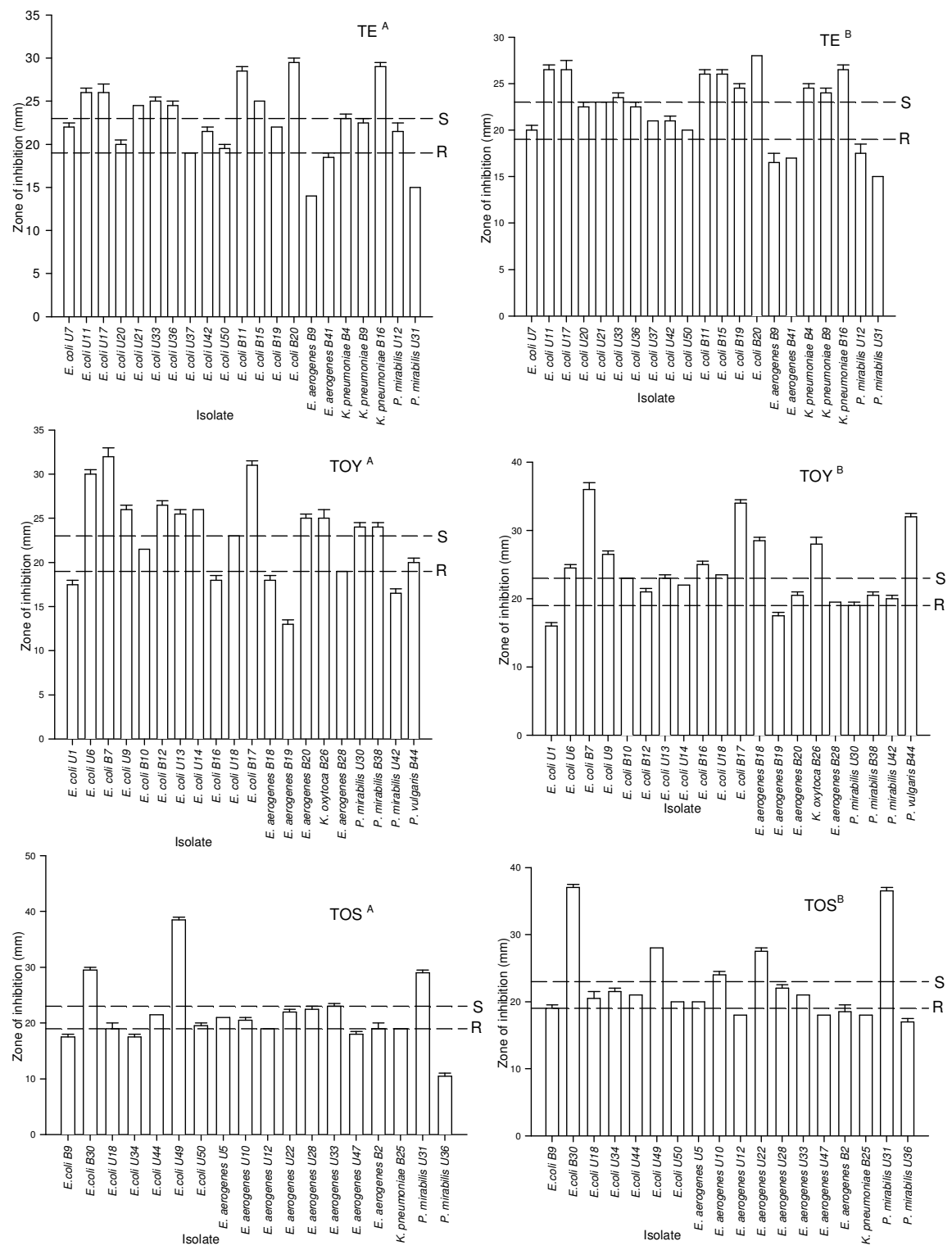

Fig. 3. Susceptibility of the isolates to meropenem and imipenem; ${ }^{A}$ represents meropenem; ${ }^{\mathrm{B}}$ represents imipenem

Nosocomial agents have the propensity to spread at an alarming rate among patients with incidence rate at about 4.5 cases per 100 people (Cunha et al., 2016; Demiraslan et al., 2017). Notably in the hereby study, Escherichia coli and Enterobacter aerogenes were the most dominant. The high frequency of $E$. coli has also been reported by Yusuf $e t$ al. (2012), Motayo et al. (2013) and Mohammed et al. (2015) in hospitals at Kano and Abeokuta, Nigeria. It can therefore be said that in this study, bacteria belonging to the Enterobacteriaceae family that are principal etiology of nosocomial infections have been isolated, which may be an indicator of an existing or impending nosocomial infection. Overall, $57.6 \%$ of the isolates were recovered from urines samples, while $42.4 \%$ were from blood samples. The higher frequency in urine samples observed was similar to the reports of $57.1 \%$ by Mohammed et al. (2015) and $52 \%$ by Lixandru et al. (2015).

\section{Antibiotic resistance of isolates}

The antibiotics used in the study belonged to different classes of antibiotics including aminoglycosides, fluoroquinolones, penicillins, nitrofurans, beta-lactam, cephalosporins and carbapenems. All the 59 Enterobacteriaceae isolates recovered from blood and urine samples in the three hospitals were multi-drug resistant. The isolates considered as most susceptible were resistant to at least 4 out of 10 antibiotics. Complete resistance to second and third- generation cephalosporins (cefuroxime and ceftazidime) was also observed in all the isolates. This observation is higher than the report of about $52.1 \%$ resistance to cephalosporins by Mohammed et al. (2015) in a hospital in Kano state, Nigeria. Resistance to cephalosporins has been reported to be an indicator of beta lactamase production and also, carbapenemase production in Enterobacteriaceae (Huang et al., 2014). 
316

According to the CLSI (2012) guidelines on the phenotypic determination of ESBL production in Enterobacteriaceae, non-susceptibility of isolates to cephalosporins (ceftazidime, ceftriaxone, cefotaxime or cefuroxime) or beta-lactam (aztreonam or amoxicillinclavulanate) could be taken as potential ESBL producers. The results of the present study are therefore suggestive that these isolates carry any of the aforementioned resistance genes.

\section{Incidence of $C R E$ and risk factor association}

The overall incidence of CRE in EKSUTH, LAUTECH Ogbomoso and LAUTECH Osogbo at the time of study from October 2016 to March 2017 was $7.7 \%$ (12.8 cases per 1,000 people). Age and exposure to invasive devices were significantly associated with CRE colonization. More so, CRE distribution was higher in patients aged 51 years and above. According to the concept of immunosenescence which explains the change in immune responses as a result of aging, individuals belonging to older age groups are more susceptible to infectious diseases, cancer development and auto immune (Castelo-Branco and Soveral, 2013; Simon et al., 2015). Montencino-Rodriguez et al. (2013) also reported that individuals that are 70 years and above have an increased vulnerability to infectious diseases. Duration of hospitalization and prolonged use of antibiotics were also factors implicated in CRE colonization. Although presence of a medical condition and surgical procedures are existing risk factors of CRE acquisition, they were not confounding factors in the hereby study. Gender of the patients was also not associated with CRE colonization.

\section{Conclusions}

The study revealed the incidence of carbapenemresistant Enterobacteriaceae (CRE) among in-patients receiving healthcare in three selected tertiary hospitals in Southwest, Nigeria. Also, the strong association of age with CRE colonization may pose high complications in older patients. Succinctly, the low incidence of CRE isolates in the study locations at the time of the survey does not nullify possible incidence in subsequent surveys. It is therefore crucial to establish a surveillance program that will ensure routine check to allow early detection of CPE in order to prevent outbreaks.

\section{References}

Albiger B, Glasner C, Struelens M, Grundmann H, Monnet D (2015). The European survey of carbapenemase-producing Enterobacteriaceae (EuSCAPE) working group-carbapenemase-producing Enterobacteriaceae in Europe: assessment by national experts from 38 countries. Euro Surveillance 20(45):2-8.

Bauer AW, Kirby WMM, Sherris JC, Turk M (1966). Antibiotic susceptibility testing by a standardized single disk method. American Journal Clinical Pathology 45:493-496.

Cai Y, Venkatachalam I, Tee N, Tan T, Kurup A, Wong S, et al, Marimuthu K (2017). Prevalence of healthcare-associated infections and antimicrobial use among adult inpatients in Singapore acute-care hospitals: Results from the first national point prevalence survey. Clinical Infectious Diseases 64:S61-S67.

Castelo-Branco C, Soveral I (2013). The immune system and aging: a review. Gynecological Endocrinology30(1):16-22.

Clinical and Laboratory Standards Institute (CLSI) (2012). CLSI Document M07-A9.

Methods for dilution antimicrobial susceptibility tests for bacteria that grow aerobically: approved standard. 19th Edition, CLSI, Wayne.

Clinical and Laboratory Standards Institute (CLSI) (2016). Performance standards for antimicrobial susceptibility testing, Twenty-sixth informational supplement. CLSI document M100-S26. Wayne, PA: Clinical and Laboratory Standards Institute.

Cunha C, Kassakian S, Chan R, Tenover F, Ziakas P, Chapin K, Mermel L (2016). Screening of nursing home residents for colonization with carbapenem-resistant Enterobacteriaceae admitted to acute care hospitals: Incidence and risk factors. American Journal of Infection Control 44(2):126-130.

Demiraslan H, Cevahir F, Berk E, Metan G, Cetin M, Alp E (2017). Is surveillance for colonization of carbapenem-resistant gram negative bacteria important in adult bone marrow transplantation units? American Journal of Infection Control 45(7):735-739.

Ejjkeugwu C, Ugwu M, Iroha I, Eze P, Gugu T, Esimone C (2014). Phenotypic detection of Metallo- $\beta$-Lactamase (MBL) enzyme in Enugu, Southeast Nigeria. American Journal of Biological, Chemical and Pharmaceutical Sciences 2(2):1-6.

European Centre for Disease and Control (ECDC) (2013). Carbapenemase-producing Enterobacteriaceae in Europe: Interim results from the European survey on carbapenemase-producing Enterobacteriaceae (EUSCAPE) project 2013. Technical Report pp 613.

Guh AY, BulensSN, Mu Y,JacobJT, RenoJ, ScottJ, et al., Kallen AJ (2015). Epidemiology of carbapenem-resistant Enterobacteriaceae in 7 US communities, 2012-2013.JAMA314(14):1479-1487.

Huang S, Liu M, Lin C, Shi Z (2014). Molecular surveillance and clinical outcomes of carbapenem-resistant Escherichia coli and Klebsiella pneumoniae infections.Journal of Microbiology, Immunology and Infection 47(3):187-196.

Jeong HJ, Jund HL, Jae JL, Kwang SP, Asad MK, Chnag-Ro L, Byeong CJ, Sang HL (2015). Structural basis for carbapenem-hydrolyzing mechanisms of carbepenemase conferring antibiotic resistance. International Journal of Molecular Science 16(5):9654-9692.

Lixandru B, Cotar A, Straut M, Usein C, Cristea D, Ciontea S, ... Damian M (2015). Carbapenemase-producing Klebsiella pneumoniae in Romania: A six-month survey. PLoSONE 10(11):e0143214.

Mohammed Y, Dabo NT, Umar MK (2015). Laboratory evaluation of clinical bacterial isolates for detection of carbapenemase-producing Enterobacteriaceae in Kano, Northwest, Nigeria. International Journal of Tropical Disease and Health 12(3):1-5.

Montecino-Rodriguez E, Berent-Maoz B, Dorshkind K (2013).Causes, consequences, and reversal of immune system aging. The Journal of Clinical Investigation 123(3):958-965.

Motayo PO, Akinduti PA, Adeyakinu FA, Okerentugba PO, Nwanze JC, Onoh CC, Innocent-Adiele HC, Okonko IO (2013). Antibiogram 
and plasmid profiling of carbapenemase and extended spectrum Betalactamase (ESBL) producing Escherichia coli and Klebsiella pneumoniae in Abeokuta, South western, Nigeria. African Health Sciences 13(4):1091-1097.

Oduyebo O, Falayi O, Oshun P, Ettu A (2015). Phenotypic determination of carbapenemase producing enterobacteriaceae isolates from clinical specimens at a tertiary hospital in Lagos, Nigeria. Nigerian Postgraduate Medical Journal 22(4):223-227.
Simon A, Hollander G, McMichael A (2015). Evolution of the immune system in humans from infancy to old age. Proceedings of the Royal Society B: Biological Sciences 282(1821):20143085.

Yusuf I, Magashi AM, Firdausi FS Sharif AA, Getso MI, Bala JA, Aliyu IA (2012). Phenotypic detection of Carbapenemases in members of Enterobacteriaceae in Kano, Nigeria. International Journal of Science and Technology 2(11):802-804 